\title{
Post Partial Nephrectomy Surveillance Imaging: an Evidence-Based Approach
}

\author{
Lorenzo Marconi • Michael A. Gorin • Mohamad E. Allaf
}

Published online: 10 March 2015

(C) Springer Science+Business Media New York 2015

\begin{abstract}
To ensure the early detection of recurrent disease, all patients should undergo routine surveillance following partial nephrectomy for renal cell carcinoma. In order to optimize resource allocation and avoid unnecessary radiation exposure, the frequency and duration of surveillance should be tailored to the individual patient's risk of cancer recurrence. The evidence for surveillance after partial nephrectomy is presented reviewing the current literature on prognostic models and proposed surveillance protocols based on the timing and patterns of renal cell carcinoma recurrence. In addition, we review recent guidelines on post partial nephrectomy surveillance as well as the literature on novel imaging techniques that may aid in early disease discovery.
\end{abstract}

Keywords Recurrent disease $\cdot$ Partial nephrectomy $\cdot$ Renal cell carcinoma $\cdot$ Radiation exposure

\section{Introduction}

The American Cancer Society estimates that approximately 60,000 new cases of renal cell carcinoma (RCC) are diagnosed annually in the United States [1]. Worldwide, this figure approaches 270,000 cases per year, with the highest incidence rates in North America and Europe [2]. Over the last three

This article is part of Topical Collection on New Imaging Techniques

L. Marconi

Urology and Renal Transplantation Department, Coimbra University

Hospital, Coimbra, Portugal

M. A. Gorin • M. E. Allaf $(\bowtie)$

The James Buchanan Brady Urological Institute and Department of

Urology, Johns Hopkins University School of Medicine, 600 North

Wolfe Street, Park 223, Baltimore, MD 21287, USA

e-mail: mallaf@jhmi.edu decades, there has been a steady increase in the incidence of clinically localized RCC. This trend has been attributed to the growing number of small renal masses incidentally detected on cross-sectional imaging performed for non-urologic conditions [3]. With this trend, treatment with partial nephrectomy (PN) has seen widespread use [4-6] as a means to avoid the sequelae of surgically induced chronic kidney disease associated with radical nephrectomy (RN) [7-10]. In fact, current guidelines from the American Urological Association (AUA) [11], National Comprehensive Cancer Network (NCCN) [12••], and European Association of Urology (EUA) [13••] now promote the use of PN whenever technically feasible for the treatment of small renal tumors. With this treatment approach, only a small minority of patients will experience a local or distant recurrence. However, due to the aggressive nature of metastatic RCC, longitudinal follow-up is essential for all patients so as to ensure the early detection and timely treatment of recurrent disease. Herein, we review the rationale and data supporting routine surveillance following PN. Special attention is paid to prognostic models and published surveillance protocols. In addition, we highlight the follow-up algorithms endorsed by the AUA, NCCN, and EAU.

\section{Rationale for Oncologic Surveillance}

In the current review, we maintain the presumption that with the use of aggressive surgical management and targeted systemic therapy, the early detection of recurrent RCC will lead to improved patient outcomes. This view is premised on the observation that a latter detected higher metastatic burden can limit the opportunity for surgical resection (now considered the standard therapy in cases of resectable and solitary metastases) [14]. Moreover, in the setting of local recurrences or metachronous tumors in the contralateral kidney, there is a limited time period for treatment with nephron-sparing 
techniques. In addition, data from clinical trials evaluating targeted therapy for metastatic RCC have consistently shown worse outcomes among patients with a greater disease burden $[15,16]$.

\section{Prognosis and Surveillance}

Postoperative surveillance should be tailored to the natural history of the disease and the magnitude of risk for cancer progression. Therefore, follow-up regimens should be based on known independent predictors of postoperative recurrence. This would avoid underscreening patients at high risk for progression and overscreening those at low risk. Such an approach would also result in improved resource allocation for the health care system and a reduction in unnecessary radiation exposure from diagnostic imaging [17].

Several approaches have been proposed to help predict the risk of RCC recurrence following surgical treatment. Anatomical stage has historically served as the most important prognostic factor for patients with RCC. Recently, however, numerous studies have demonstrated that a combination of clinical and histopathological factors can better predict tumor recurrence than that of stage alone. We present some examples of prognostic models which can be used to establish appropriate patient-tailored surveillance protocols:

Kattan et al. (MSKCC) [18] designed a nomogram to predict 5-year recurrence-free survival based on prospectively collected data from 601 patients submitted to RN or PN. The predictors of recurrence were symptoms at presentation (local vs. systemic), histologic subtype (clear cell vs. papillary vs. chromophobe), tumor size, and pT stage (AJCC 1997). Multivariate analysis suggested no independent effect of the type of surgery (RN vs. PN) on recurrence rate. An updated postoperative nomogram from this same group [19] focused on the clear cell variant only, including the following predictors: size, pT stage (AJCC 2002), Fuhrman grade, necrosis, microvascular invasion, and symptom presentation.

Leibovich and colleagues [20] from the Mayo Clinic developed an alternative algorithm to predict progression to metastatic RCC in patients with clinically localized clear cell RCC after $\mathrm{RN}$ (at 1, 3, 5, 7, and 10 years). The features in this model included $\mathrm{pT}$ and $\mathrm{pN}$ stage (2002 AJCC), tumor size ( $<10 \mathrm{vs.}$ $\geq 10 \mathrm{~cm}$ ), nuclear grade, and the presence of tumor necrosis. This same group retrospectively identified 1864 patients who underwent PN (12\%) or RN (88 \%) for non-metastatic RCC [21]. Using multivariate analysis, four scoring algorithms were proposed to predict disease recurrence at various time points (0.25-10 years) in specific regions of the body (abdomen, thorax, bone, and brain) [21]. Based on the patterns of recurrence in the various risk groups, the authors argued that patients with higher scores should be followed closely in the first 2 to 3 years and require less vigorous follow-up thereafter.
Patients with moderate scores require continuous follow-up even after the first 5 years, and patients with low scores would require minimal to no follow-up. The retrospective nature of this study and the variation in the follow-up protocol of the patients included may have led to underestimation of disease recurrence rates, prompting caution in the immediate translation of the results in a surveillance protocol. Routine screening for brain or bone metastasis was not advocated due to their symptomatic nature.

A third prognostic model known as The UCLA Integrated Staging System (UISS) was developed by Ziman and coworkers [22] and incorporates tumor node metastasis (TNM) stage (AJCC 1997), Fuhrman Grade, and Eastern Cooperative Oncology Group performance status to stratify patients according to their probability of survival and tumor recurrence. Based on the UISS staging system, a postoperative surveillance protocol was created [23].

The above cited prognostic models have been externally validated with performances ranging between 74 and $82 \%$ [24]. However, due to the wide variability in the timing and pattern of treatment failure within risk groups, additional work is needed to better risk stratify patients. One hope lies in the development of novel biomarkers such as circulating tumor cells and circulating tumor DNA $[25,26]$.

\section{Review of Published Surveillance Protocols}

By analyzing the timing and patterns of RCC recurrence, several surveillance protocols have been proposed [23, 27-33]:

Sandock et al. [31] retrospectively reviewed 137 patients with localized RCC, submitted to RN, and followed for 14 years. The average interval and site of RCC recurrence were analyzed for each TNM stage (AJCC 1992). Disease recurred in $0,14.6$, and $52.8 \%$ of patients with pT1, pT2, and pT3, respectively. Among patients with pT2-3, $85 \%$ of recurrences occurred within the first 3 years postoperatively. The remaining $15 \%$ recurred between 3.4 and 11.4 years. The most common sites of recurrence were the lung, liver, bone, and brain. Notably, $73.7 \%$ of pulmonary metastases were symptomatic and $100 \%$ of patients had an abnormal chest X-ray (CXR). Regarding abdominal metastasis, $92.3 \%$ of patients had symptoms or abnormal serum studies. Based on these data, the authors recommended against routine radiographic follow-up of patients with pT1 RCC (defined as $\leq 2.5 \mathrm{~cm}$ by the AJCC 1992). In contrast, for patients with pT2-3 disease, they recommended a CXR along with history and physical exam every 6 months postoperatively for 3 years and then yearly thereafter. In terms of imaging the abdomen, bone, and brain, the authors recommended that computed tomography (CT) be performed only for a suspicion of recurrence. 
In a similar study, Hafez et al. [28] reviewed 327 cases of PN performed at the Cleveland Clinic and found that 38 patients $(11.6 \%)$ experienced a recurrence during a mean follow-up of 55.6 months. Among these cases, 13 (34.3\%) recurred locally ( 7 of them with metachronous metastatic disease). Recurrent RCC was detected by symptoms in 25 (65.8\%) patients and by follow-up CXR or abdominal CT in $13(34.3 \%)$. Notably, no patient with pT1 RCC (defined as $\leq 2.5 \mathrm{~cm}$ by the AJCC 1992) experienced a local recurrence and only 3 (4.4\%) developed metastatic disease. Given these findings, the authors recommended against radiographic surveillance of patients with $\mathrm{pT} 1$ disease. Of patients with $\mathrm{pT} 2$ RCC (defined as $>2.5 \mathrm{~cm}$ limited to the kidney by the AJCC 1992), $2 \%$ experienced a local recurrence (detected after more than 4 years) and $5.3 \%$ developed metastases (the lung being the initial and most common site of metastasis). These data revealed that although frequent early radiographic monitoring for local tumor recurrence was likely unnecessary, abdominal $\mathrm{CT}$ every 2 years was an important measure. A yearly CXR was recommended in this group. Lastly, among patients with pT3 RCC (invading perinephric fat/renal venous system) treated with $\mathrm{PN}$, the authors observed a local recurrence rate of $9.3 \%$ and metastatic recurrence rate of $13 \%$. Seventy percent of the local recurrences were diagnosed within the first 2 years. The most common site of metastasis was the lung. In light of these data, the authors recommended a biannual abdominal CT in the first 2 years and every 2 years thereafter, along with annual CXR.

In a departure from risk stratification using standard clinicopathologic variables, Ljungberg et al. [30] recommended a follow-up protocol based on the DNA ploidy status (diploid vs. aneuploid) of the patient's tumor. These authors recommended against surveillance for patients with diploid pT1T2 tumors or with aneuploid pT1 tumors $<5 \mathrm{~cm}$ (AJCC 1997). In contrast, the authors recommended that patients with aneuploid pT1-T2 tumors $>5 \mathrm{~cm}$ should be followed with biannual CXR. Additionally, for those with pT3 tumors, abdominal, CT was recommended at 6 and 12 months postoperatively.

An innovative surveillance protocol tailored to the UISS risk groups was presented by Lam et al. [23]. In this report, a cohort of 559 patients surgically treated for RCC (30\% with PN) was divided into four risk groups. Patients in the low risk group had a recurrence rate of $<10 \%$ and a long time to recurrence (median 28.9 months). The majority of recurrences occurred in the lungs and one third occurred in the first year (median 23.6 months), supporting the use of yearly chest CT in these patients. The median time of abdominal recurrences was 32 months and an abdominal CT was recommended at 24 and 48 months. Surveillance after 5 years was not recommended. Intermediate-risk group patients had a higher recurrence rate at $32 \%$. The majority of recurrences occurred in the chest (74.4\%) and abdomen (58.1\%), and 41.7 and $58.1 \%$ were diagnosed in the first year of follow-up, respectively. Based on these data, chest $\mathrm{CT}$ scans were recommended every 6 months until 3 years and yearly thereafter. Abdominal CT should be done at 1 year and every 2 years thereafter. In the high-risk group, chest and abdominal recurrences were frequent and often occurred within the first 6 months; thus, the authors recommended serial chest CT scans every 6 months, continuing for 3 years and yearly thereafter (after 3 years alternating CXR and chest CT is acceptable). In addition, they recommended that an abdominal CT should be done every 6 months for 2 years and yearly thereafter. Given a 7.1 and $5 \%$ incidence of late chest and abdominal recurrences, it was recommended that patients be followed beyond this time with less frequent intervals. Patients with nodal disease presented earlier and with more aggressive recurrences. Therefore, for this group, the authors recommended that chest and abdominal CT be performed at 3, 6, 12, 18, and 24 months. A yearly chest and abdominal CT scan would be sufficient thereafter. The authors concluded that using this staged oriented protocol, patients should be followed similarly after PN or RN. A more recent follow-up scheme was developed by Antonelli et al. [27] based on the time and patterns of RCC recurrence of 814 patients after RN and PN (20\%).

Siddiqui and coworkers [32] reported the first histologic subtype specific surveillance algorithm. After reviewing 2339 patients submitted to RN or PN (15\%) at the Mayo Clinic, a Cox proportional hazard model was used to determine which further pathological features were independently predictive of abdominal and thoracic recurrence in each one of three groups (clear cell, papillary, and chromophobe). For clear cell RCC, the surveillance algorithm included the following features: surgical margin, pT, pN (2002 AJCC), tumor size ( $<10 \mathrm{vs.} \geq 10 \mathrm{~cm}$ ), nuclear grade, and presence of necrosis. For papillary and chromophobe RCC, stage and grade were the only predictors of recurrence included in the algorithm. A decrease in recurrence-free survival superior to $1 \%$ over a specific period (periods of 3 months during the first 3 years and yearly periods thereafter) would trigger a sitespecific imaging test. Postoperative imaging was tailored for each histologic subtype and recurrence risk group.

\section{Ideal Duration of Follow-up}

The greatest risk of recurrence for patients with RCC exists in the first 5 years following nephrectomy [30,31]. In a series of 187 patients treated with RN, $43 \%$ of metastases developed within 1 year, $70 \%$ within 2 years, $80 \%$ within 3 years, and $93 \%$ by the end of 5 years of follow-up [30]. Based on these and similar data, it is widely held that follow-up should be most intense during the first 3 years following surgery, with a decrease in the frequency of surveillance in subsequent years. It is worth noting, however, that up to $10 \%$ of the 
patients may develop late recurrences beyond 5 years after surgery. At the present time, however, it remains difficult to know which patients would most benefit from imaging beyond 5 years of follow-up.

One study found that the development of late recurrence was associated with lymphovascular invasion, high Fuhrman grade (III-IV), and pathologic tumor stage $>$ pT1 at primary diagnosis [34]. In a related report, Adamy et al. [35] compared patients with late recurrence to those with early recurrence after nephrectomy. The late recurrence group patients had less aggressive disease and were more likely to present asymptomatically. Of note, the lung and bones were the most frequent late metastatic sites. However, a significant number of patients with late recurrences were noted at unusual sites such as the pancreas, contralateral adrenal gland, and thyroid. This observation underscores the need to maintain a high suspicion for RCC recurrence in patients presenting history of nephrectomy and a new lesion regardless of the affected site and the diseasefree interval

It is worth noting that positive surgical margins after PN seem to harbor an increased risk of disease recurrence, especially in patients with tumors of high malignant potential [36]. Thus, these patients require special consideration when determining the frequency and duration of follow-up. With that said, the vast majority of patients with a positive surgical margin will not experience local or distant tumor recurrence. Clearly, additional data is needed in order to determine the optimal follow-up protocol for this patient cohort.

\section{Surveillance Protocols Endorsed by the AUA, NCCN, and EAU}

AUA In 2013, the AUA published guidelines on the surveillance of patients with clinically localized RCC [37••]. Contained within this document are specific guidelines for patients treated with PN. For those at low risk of recurrence (defined as pT1 RCC), the AUA recommends baseline abdominal imaging with either CT or MRI at 3 to 12 months postoperatively. For 3 years thereafter, patients may undergo annual surveillance with CT, MRI, or ultrasound (US) of the abdomen as well as CXR. For patients at moderate to high risk of recurrence (defined at $\geq \mathrm{pT} 2 \mathrm{RCC}$ ), the AUA recommends cross-sectional imaging of the chest and abdomen at 3 to 6 months following surgery. It is further recommended that patients undergo abdominal and chest imaging every 6 months for at least 3 years and then annually thereafter to year 5 . Follow-up imaging may be performed with a combination of ultrasound (US), CXR, CT, and/or MRI.

NCCN The NCCN [12••] recommends baseline abdominal imaging with CT, MRI, or US within 3 to 12 months of surgery for patients with pT1 RCC. Following initial postoperative imaging, three additional years of annual abdominal and chest imaging is recommended. Chest imaging may be performed with either CXR or CT. For the subset of patients with $\geq p T 2 \mathrm{RCC}$, more rigorous follow-up is recommended with chest and abdominal imaging every 3 to 6 months for 3 years and then annually for two additional years.

EAU The 2014 guideline from the EAU [13••] proposes an algorithm for surveillance-based risk stratification using one of the validated classification systems (e.g., UISS). Following a baseline US, it is recommended that patients with a low-risk profile be imaged yearly, alternating US with chest/abdominal $\mathrm{CT}$ for the next 5 years. For patients at intermediate to high risk for recurrence, annual imaging with a chest and abdominal CT is recommended for 5 years and then biennially thereafter.

Table 1 summarizes the guidelines from the AUA, NCCN, and EAU. In addition, this table details the frequency of follow-up by history, physical exam, and laboratory testing.

To date, only one report has evaluated the efficacy of the proposed surveillance guidelines. In this study, Stewart et al.[38] assessed the ability of the AUA 2013 and NCCN 2014 protocols to capture RCC recurrences and determined the duration of surveillance required to capture 90,95 , and $100 \%$ of recurrences. This retrospective analysis of 3651 patients who underwent RN or PN (27.1\%), with a median follow-up of 9 years, revealed disease recurrence in 1088 patients. The overall recurrence detection rate, using the AUA 2013 and the NCCN 2014 protocols, were 66.9 and $68.2 \%$, respectively. In the low-risk PN group (pT1N0), only 37.2 and $38.3 \%$ of recurrences would have been captured if the AUA 2013 and NCCN 2014 schemes were followed. Better results would be achieved with the moderate/high risk( $>$ pT1 or positive margin) surveillance protocols with a detection rate of about $80 \%$. This study revealed that to capture $95 \%$ of recurrences among low risk PN patients, follow-up would be required for 15 years for the chest, 11 years for abdomen, 5 years for bone, and 11 years for other sites.

\section{Novel Imaging Modalities}

As outlined above, current guidelines support the use of CXR, US, and cross-sectional imaging for the surveillance of patients following PN. The sensitivity of these conventional imaging modalities, however, are limited to target lesion sizes $>1 \mathrm{~cm}$ in diameter. Molecular imaging with positron emission tomography (PET) offers superior spatial resolution and thus the promise of earlier disease detection. At present, however, the use of PET-based imaging remains experimental in the setting of RCC and is not recommended in routine clinical practice. 


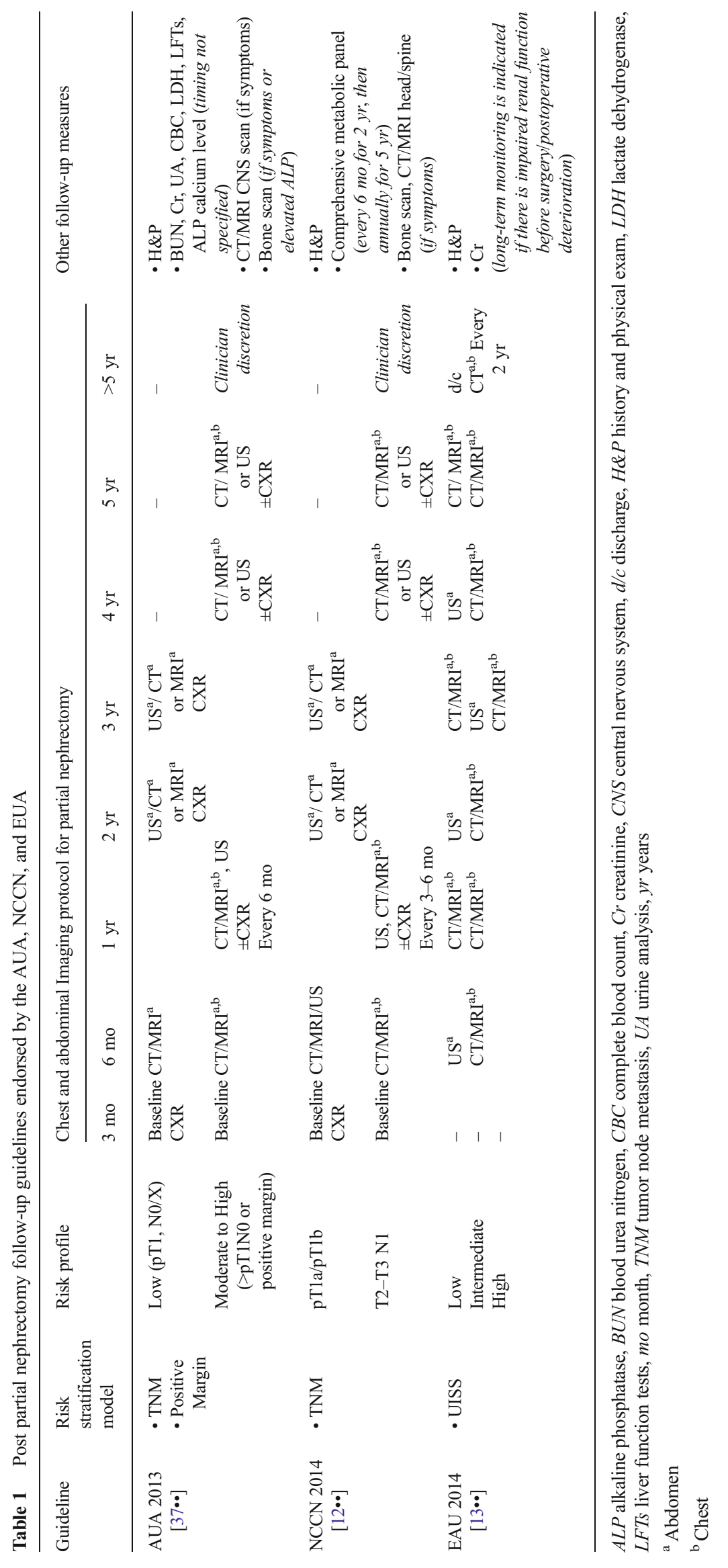


The use of fluorodeoxyglucose (FDG) to functionally image malignancies is based on the anticipated altered glycolytic pathway in malignant cells (i.e., the Warburg effect). This technique has the potential to characterize RCC at the cellular and subcellular level in a non-invasive fashion. When used in combination with $\mathrm{CT}$, it provides functional and anatomic tumor characteristics. Despite the unfavorable performance of FDG-PET in diagnosing primary renal tumors, FDG-PET may be helpful in the evaluation of recurrent RCC. In a recent meta-analysis of the diagnostic accuracy of FDG-PET to determine the presence of malignancy within the kidney and extra renal sites, the pooled sensitivity and specificity were 79 and $90 \%$ [39]. These results show a better accuracy to detect extra renal RCC possibly because these lesions are not obscured by background urinary FDG activity. It seems that FDG-PET imaging may not localize to small lesions as accurately as larger ones. In fact, Majhail et al. [40] showed that the sensitivity of FDG-PET increased from $63.6 \%$ when considering all lesions to $92.9 \%$ for lesions with size superior to $2 \mathrm{~cm}$. Some studies have shown that FDG-PET may localize high-grade tumors more efficiently than low-grade tumors [41].

Nakatani et al. [42] studied the value of FDG-PET to detect recurrent and distant metastatic disease in 23 patients that had previous nephrectomy. Overall, the sensitivity, specificity, and diagnostic accuracy of FDG-PET for detecting recurrent malignancy were 81,71 , and $79 \%$, respectively. There were four false negative results (one case of lung and brain metastasis, one case of multiple small liver metastasis, and two cases of metastasis to the pancreas). The findings were false positive in two inflammatory chest lesions. This retrospective study was affected by patient selection bias that may have overestimated the overall accuracy. In fact FDG-PET imaging has the inherent limitation of being non-cancer specific, resulting in the accumulation of FDG in many benign tissues (infection, inflammation, and trauma) and normal tissues that have a physiological propensity to accumulate FDG (kidneys, ureters, and bladder).

Using antibodies that have highly selective affinity to cancer-specific antigens, immuno-PET offers an exciting strategy to image various types of cancers, particularly RCC. The chimeric antibody cG250 (girentuximab) binds with carbonic anhydrase IX, a cell surface antigen highly and homogeneously expressed in more than $95 \%$ of clear cell RCC. Divgi et al. [43-] have shown in a multicentric phase III clinical trial (REDECT) that ${ }^{124}$ I-girentuximab PET/CT can accurately identify localized clear cell RCC with higher sensitivity and specificity to contrast enhanced CT. In the setting of metastatic disease, ${ }^{124}$ I-girentuximab PET has been shown to identify anatomically occult regional node metastasis [44]. Furthermore, conventional scintigraphy showed high and specific uptake of ${ }^{111}$ In-cG250 in metastatic lesions, including lung lesions, considered difficult to visualize with ${ }^{131} \mathrm{I}-\mathrm{cG} 250$
[45]. The advantage of PET over conventional scintigraphy is the higher spatial resolution and the ability to accurately determine antibody uptake in tissues [46].

\section{Conclusion}

To ensure the early detection of recurrent disease, all patients should undergo routine surveillance following PN. The frequency and duration of follow-up should be tailored to the individual patient's risk for recurrence. Guidelines from the AUA, NCCN, and EAU provide guidance for the surveillance of this patient population. These recommendations, however, are based on data from retrospective studies. Thus, additional well-designed studies are needed to more precisely define the optimal surveillance of patients following PN. Furthermore, future work should aim to incorporate molecular imaging and novel biomarkers capable of detecting early recurrences which may otherwise be unapparent with conventional imaging techniques.

\section{Compliance with ethics guidelines}

Conflict of interest Dr. Lorenzo Marconi, Dr. Michael A. Gorin, and Dr. Mohamad E. Allaf each declare no potential conflicts of interest

Human and animal rights and informed consent This article does not contain any studies with human or animal subjects performed by any of the authors.

\section{References}

Papers of particular interest, published recently, have been highlighted as:

- Of importance

•. Of major importance

1. Siegel R, Ma J, Zou Z, Jemal A. Cancer statistics, 2014. CA: Cancer J Clin. 2014;64:9-29.

2. Ferlay J, Shin HR, Bray F, Forman D, Mathers C, Parkin DM. Estimates of worldwide burden of cancer in 2008: GLOBOCAN 2008. Int J Cancer J Int du Cancer. 2010;127:2893-917.

3. Gill IS, Aron M, Gervais DA, Jewett MA. Clinical practice. Small renal mass. N Engl J Med. 2010;362:624-34.

4. Miller DC, Saigal CS, Banerjee M, Hanley J, Litwin MS. Urologic Diseases in America P. Diffusion of surgical innovation among patients with kidney cancer. Cancer. 2008;112:1708-17.

5. Woldrich JM, Palazzi K, Stroup SP, Sur RL, Parsons JK, Chang D, et al. Trends in the surgical management of localized renal masses: thermal ablation, partial and radical nephrectomy in the USA, 1998-2008. BJU Int. 2013;111:1261-8.

6. Poon SA, Silberstein JL, Chen LY, Ehdaie B, Kim PH, Russo P. Trends in partial and radical nephrectomy: an analysis of case logs from certifying urologists. J Urol. 2013;190:464-9. 
7. Weight CJ, Larson BT, Fergany AF, Gao T, Lane BR, Campbell $\mathrm{SC}$, et al. Nephrectomy induced chronic renal insufficiency is associated with increased risk of cardiovascular death and death from any cause in patients with localized cT1b renal masses. J Urol. 2010;183:1317-23.

8. Kim SP, Thompson RH, Boorjian SA, Weight CJ, Han LC, Murad $\mathrm{MH}$, et al. Comparative effectiveness for survival and renal function of partial and radical nephrectomy for localized renal tumors: a systematic review and meta-analysis. J Urol. 2012;188:51-7.

9. Mariusdottir E, Jonsson E, Marteinsson VT, Sigurdsson MI, Gudbjartsson T. Kidney function following partial or radical nephrectomy for renal cell carcinoma: a population-based study. Scand J Urol. 2013;47:476-82.

10. Yap SA, Finelli A, Urbach DR, Tomlinson GA, Alibhai SM. Partial Nephrectomy for the Treatment of Renal Cell Carcinoma and the Risk of End Stage Renal Disease. BJU Int. 2014.

11. Campbell SC, Novick AC, Belldegrun A, Blute ML, Chow GK, Derweesh $\mathrm{IH}$, et al. Guideline for management of the clinical T1 renal mass. J Urol. 2009;182:1271-9.

12.• Motzer RJE, Agarwal N, Beard C, Bhayani S, Bolger G, Chang S, Choueiri T, Derweesh I, Gupta S, Hancock S, Kim J, Kuzel T, Lam E, Lau C, Levine E, Lin D, Michaelson D, Olencki T, Pili R, Plimack E, Rampersaud E, Redman B, Ryan C, Sheinfeld J, Sircar K, Somer B, Wilder R. NCCN Guideline Kidney Cancer. http://www.nccn.org/professionals/physician_gls/f_guidelines_ nojava.asp-site. 2014. Current gudeline from the NCCN on the managment of renal cell carcinoma.

13.• Ljungberg BBK, Bex A, Canfield S, Dabestani S, Hofmann F, Hora M, Kuczyk M, Lam T, Marconi L, Merseburger L, Mulders P, Powles T, Staehler M, Volpe A, Renal Cell Cancer Guidelines. 2014. The 2014 guideline from the EAU proposes an algorithm for surveillance based risk-stratification using one of the validated classification systems.

14. Dabestani S, Marconi L, Hofmann FSF, Lam T, Canfield S, Staehler $\mathrm{M}$, et al. Local treatments for metastases of renal cell carcinoma: a systematic review. Lancet Oncol. 2014;15:e549-61.

15. Escudier B, Michaelson MD, Motzer RJ, Hutson TE, Clark JI, Lim $\mathrm{HY}$, et al. Axitinib versus sorafenib in advanced renal cell carcinoma: subanalyses by prior therapy from a randomised phase III trial. Br J Cancer. 2014;110:2821-8.

16. Motzer RJ, Hutson TE, Tomczak P, Michaelson MD, Bukowski RM, Oudard S, et al. Overall survival and updated results for sunitinib compared with interferon alfa in patients with metastatic renal cell carcinoma. J Clin Oncol: Off J Am Soc Clin Oncol. 2009;27: 3584-90

17. Lipsky MJ, Shapiro EY, Hruby GW, McKiernan JM. Diagnostic radiation exposure during surveillance in patients with pTla renal cell carcinoma. Urology. 2013;81:1190-5.

18. Kattan MW, Reuter V, Motzer RJ, Katz J, Russo P. A postoperative prognostic nomogram for renal cell carcinoma. J Urol. 2001;166: 63-7.

19. Sorbellini M, Kattan MW, Snyder ME, Reuter V, Motzer R, Goetzl $\mathrm{M}$, et al. A postoperative prognostic nomogram predicting recurrence for patients with conventional clear cell renal cell carcinoma. J Urol. 2005; 173:48-51.

20. Leibovich BC, Blute ML, Cheville JC, Lohse CM, Frank I, Kwon ED, et al. Prediction of progression after radical nephrectomy for patients with clear cell renal cell carcinoma: a stratification tool for prospective clinical trials. Cancer. 2003;97:1663-71.

21. Frank I, Blute ML, Cheville JC, Lohse CM, Weaver AL, Leibovich $\mathrm{BC}$, et al. A multifactorial postoperative surveillance model for patients with surgically treated clear cell renal cell carcinoma. J Urol. 2003;170:2225-32.

22. Zisman A, Pantuck AJ, Wieder J, Chao DH, Dorey F, Said JW, et al. Risk group assessment and clinical outcome algorithm to predict the natural history of patients with surgically resected renal cell carcinoma. J Clin Oncol: Off J Am Soc Clin Oncol. 2002;20: 4559-66.

23. Lam JS, Shvarts O, Leppert JT, Pantuck AJ, Figlin RA, Belldegrun AS. Postoperative surveillance protocol for patients with localized and locally advanced renal cell carcinoma based on a validated prognostic nomogram and risk group stratification system. J Urol. 2005;174:466-72. discussion 72; quiz 801.

24. Sun M, Shariat SF, Cheng C, Ficarra V, Murai M, Oudard S, et al. Prognostic factors and predictive models in renal cell carcinoma: a contemporary review. Eur Urol. 2011;60:644-61.

25. Heidenreich A, Schrader AJ, Varga Z. Basic science and research in renal cell carcinoma: from workbench to bedside. Curr Opin Urol. 2003;13:457-62.

26. Small AC, Gong Y, Oh WK, Hall SJ, van Rijn CJ, Galsky MD. The emerging role of circulating tumor cell detection in genitourinary cancer. J Urol. 2012;188:21-6.

27. Antonelli A, Cozzoli A, Zani D, Zanotelli T, Nicolai M, Cunico SC, et al. The follow-up management of non-metastatic renal cell carcinoma: definition of a surveillance protocol. BJU Int. 2007;99: 296-300.

28. Hafez KS, Novick AC, Campbell SC. Patterns of tumor recurrence and guidelines for followup after nephron sparing surgery for sporadic renal cell carcinoma. J Urol. 1997;157:2067-70.

29. Levy DA, Slaton JW, Swanson DA, Dinney CP. Stage specific guidelines for surveillance after radical nephrectomy for local renal cell carcinoma. J Urol. 1998;159:1163-7.

30. Ljungberg B, Alamdari FI, Rasmuson T, Roos G. Follow-up guidelines for nonmetastatic renal cell carcinoma based on the occurrence of metastases after radical nephrectomy. BJU Int. 1999;84:405-11.

31. Sandock DS, Seftel AD, Resnick MI. A new protocol for the followup of renal cell carcinoma based on pathological stage. J Urol. 1995; 154:28-31.

32. Siddiqui SA, Frank I, Cheville JC, Lohse CM, Leibovich BC, Blute ML. Postoperative surveillance for renal cell carcinoma: a multifactorial histological subtype specific protocol. BJU Int. 2009;104: 778-85.

33. Stephenson AJ, Chetner MP, Rourke K, Gleave ME, Signaevsky M, Palmer B, et al. Guidelines for the surveillance of localized renal cell carcinoma based on the patterns of relapse after nephrectomy. $\mathrm{J}$ Urol. 2004;172:58-62.

34. Brookman-May S, May M, Shariat SF, Xylinas E, Stief C, Zigeuner $\mathrm{R}$, et al. Features associated with recurrence beyond 5 years after nephrectomy and nephron-sparing surgery for renal cell carcinoma: development and internal validation of a risk model (PRELANE score) to predict late recurrence based on a large multicenter database (CORONA/SATURN Project). Eur Urol. 2013;64:472-7.

35. Adamy A, Chong KT, Chade D, Costaras J, Russo G, Kaag MG, et al. Clinical characteristics and outcomes of patients with recurrence 5 years after nephrectomy for localized renal cell carcinoma. $\mathrm{J}$ Urol. 2011;185:433-8.

36. Khalifeh A, Kaouk JH, Bhayani S, Rogers C, Stifelman M, Tanagho YS, et al. Positive surgical margins in robot-assisted partial nephrectomy: a multi-institutional analysis of oncologic outcomes (leave no tumor behind). J Urol. 2013;190:1674-9.

37.• Donat SM, Bishoff JT, Coleman JA, Dahm P, Derweesh IH, Herrell SD III, Hilton S, Jonasch E, Lin DW, Reuter VE, Chang SS. Follow-up for clinically localized renal neoplasms: AUA guideline. 2013. The AUA guidelines on the surveillance of patients with clinically-localized RCC after treatment with surgery or ablative procedures. The recommendations are based on a well conducted systematic review of the literature.

38. Stewart F, Thompson H, Psutka S, Cheville J, Lohse C, Boorjian S, Leibovich B. Evaluation of the National Comprehensive Cancer Network and American Urological Association Renal Cell Carcinoma Surveillance Guidelines. J Clin Oncol. 2014. in press. 
39. Wang HY, Ding HJ, Chen JH, Chao CH, Lu YY, Lin WY, et al. Meta-analysis of the diagnostic performance of [18 F]FDG-PET and PET/CT in renal cell carcinoma. Cancer Imaging : Off Publ Int Cancer Imaging Soc. 2012;12:464-74.

40. Majhail NS, Urbain JL, Albani JM, Kanvinde MH, Rice TW, Novick AC, et al. F-18 fluorodeoxyglucose positron emission tomography in the evaluation of distant metastases from renal cell carcinoma. J Clin Oncol: Off J Am Soc Clin Oncol. 2003;21: 3995-4000.

41. Kumar R, Shandal V, Shamim SA, Jeph S, Singh H, Malhotra A. Role of FDG PET-CT in recurrent renal cell carcinoma. Nucl Med Commun. 2010;31:844-50.

42. Nakatani K, Nakamoto Y, Saga T, Higashi T, Togashi K. The potential clinical value of FDG-PET for recurrent renal cell carcinoma. Eur J Radiol. 2011;79:29-35.

43. Divgi CR, Uzzo RG, Gatsonis C, Bartz R, Treutner S, Yu JQ, et al. Positron emission tomography/computed tomography identification of clear cell renal cell carcinoma: results from the REDECT trial. J Clin Oncol: Off J Am Soc Clin Oncol. 2013;31:187-94. A prospective fully-paired direct comparison of girentuximab PET/ $C T$ to multiphasic contrast-enhanced CT to characterize renal masses.

44. Povoski SP, Hall NC, Murrey Jr DA, Sharp DS, Hitchcock CL, Mojzisik CM, et al. Multimodal imaging and detection strategy with 124 I-labeled chimeric monoclonal antibody cG250 for accurate localization and confirmation of extent of disease during laparoscopic and open surgical resection of clear cell renal cell carcinoma. Surg Innov. 2013;20:59-69.

45. Stillebroer AB, Boerman OC, Desar IM, Boers-Sonderen MJ, van Herpen CM, Langenhuijsen JF, et al. Phase 1 radioimmunotherapy study with lutetium 177-labeled anti-carbonic anhydrase IX monoclonal antibody girentuximab in patients with advanced renal cell carcinoma. Eur Urol. 2013;64:478-85.

46. Juweid ME, Cheson BD. Positron-emission tomography and assessment of cancer therapy. N Engl J Med. 2006;354: 496-507. 